\title{
A Combined Softening and Hardening Mechanism for Low Frequency Human Motion Energy Harvesting Application
}

\author{
Khalis Suhaimi, Roszaidi Ramlan, and Azma Putra \\ Center for Advanced Research on Energy (CARE), Faculty of Mechanical Engineering, Universiti Teknikal Malaysia Melaka, \\ Hang Tuah Jaya, 76100 Durian Tunggal, Melaka, Malaysia
}

Correspondence should be addressed to Roszaidi Ramlan; roszaidi@utem.edu.my

Received 30 May 2014; Accepted 13 August 2014; Published 7 September 2014

Academic Editor: Marek Pawelczyk

Copyright (C) 2014 Khalis Suhaimi et al. This is an open access article distributed under the Creative Commons Attribution License, which permits unrestricted use, distribution, and reproduction in any medium, provided the original work is properly cited.

This paper concerns the mechanism for harvesting energy from human body motion. The vibration signal from human body motion during walking and jogging was first measured using 3-axes vibration recorder placed at various places on the human body. The measured signal was then processed using Fourier series to investigate its frequency content. A mechanism was proposed to harvest the energy from the low frequency-low amplitude human motion. This mechanism consists of the combined nonlinear hardening and softening mechanism which was aimed at widening the bandwidth as well as amplifying the low human motion frequency. This was realized by using a translation-to-rotary mechanism which converts the translation motion of the human motion into the rotational motion. The nonlinearity in the system was realized by introducing a winding spring stiffness and the magnetic stiffness. Quasi-static and dynamic measurement were conducted to investigate the performance of the mechanism. The results show that, with the right degree of nonlinearity, the two modes can be combined together to produce a wide flat response. For the frequency amplification, the mechanism manages to increase the frequency by around 8 times in terms of rotational speed.

\section{Introduction}

The significant reduction in power consumption to operate mobile gadgets and wireless sensors has induced vast research interest in harvesting energy from ambient sources. Scavenging energy from ambient vibration is one of the examples. Most vibration based scavenging device is traditionally configured as a linear resonant generator that consists of a single degree of freedom (SDOF) mass-spring-damper system. This particular generator is particularly useful for high frequency applications as well as when the ambient frequency does not vary with time. The optimum power of the linear resonant generator can only be obtained when the natural frequency of the device matches the excitation frequency. A Slight mismatch between these two frequencies may lead to a large reduction in the power harvested [1].

Researchers have introduced tuning techniques into the generator so that the natural frequency of the device can be changed according to the input frequency. Eichhorn et al. [2] used a prestress mechanism that can passively be adjusted by rotating a screw to alter the device natural frequency. An active tuning method proposed by Zhu et al. [3] utilized a microcontroller that can adjust the separation of interacting magnets to alter its natural frequency. However, active tuning may induce complex tuning scheme and requires power during the tuning process. On the other hand, Sari et al. [4], Shahruz [5], and Xue et al. [6] proposed multimodal generators which consist of multiple arrays of beams with different stiffness thus resulting in a different natural frequency for each beam.

Recently, nonlinear harvesters have become a popular choice among the researchers. Typical nonlinear harvesters are normally categorized into three types and they are hardening mode, softening mode, and bistable mode. These three modes of nonlinear harvester are normally known as the Duffing oscillator. Nonlinear harvesters are becoming more popular because they do not require any additional tuning mechanism to be integrated in the device and this tuning ability is already embedded in the device such as with the use of a nonlinear spring. The studies on the hardening mode have been conducted theoretically $[7,8]$ and experimentally [9-11]. The bistable mode has also been investigated by several researchers including theoretical studies $[12,13]$ as well as experimental studies $[11,14,15]$ to characterize the behaviour 
of such mode. These particular studies on hardening and bistable modes showed that the device employing these types of nonlinearities is less sensitive to a range of excitation frequency, thus making them suitable for applications with varying frequency. The softening mode, on the other hand, has been investigated by Stanton et al. [16] and Nguyen and Halvorsen [17]. In particular, Nguyen and Halvorsen [17] have concluded that their proposed device was able to perform better in the region below the natural frequency of the device showing the ability of the softening mode in amplifying the level of input.

Human motion can be regarded as the low frequency ambient sources. Thus, a standard linear resonant generator is also of limited use since the power harvested depends also on the cube of excitation frequency. To overcome these limitations, researchers also introduced the frequency-up conversion into the device. Zhu et al. [18] have proposed an energy harvester that benefits the force from the foot strike. Pillatsch et al. [19] utilized a mechanism of oscillating proof mass that is able to produce peak power scavenged at input of $2 \mathrm{~Hz}$, while Wei et al. [20] and $\mathrm{Gu}$ and Livermore [21] have considered an impact-driven frequency-up conversion method into their devices. However, the use of nonlinear softening and hardening mechanism in application involving human body motion has not been greatly explored especially involving a mechanism with a combination of hardening and softening modes.

This paper presents some experimental results of the combined nonlinear softening and hardening modes for harvesting energy from low frequency human body motion. The mechanism proposed in this paper utilizes the translationto-rotary method. The motion of the base is picked up by two oscillating masses which then rotate the $70 \mathrm{~mm}$ diameter flywheel. The amplification of the frequency is realized in terms of final rotational speed by meshing the flywheel with a $12 \mathrm{~mm}$ diameter amplification gear. In the combined mode, the softening mode is aimed at contributing to the amplification of the small amplitude-low frequency input to higher amplitude. This amplification in the amplitude results in the increase in rotational speed thus increasing the output frequency. The hardening mode, on the other hand, is aimed at widening the range of frequency over which the device is insensitive to the excitation frequency. Hence the combined softening and hardening mode is hoped to produce a mechanism which is able to amplify the small amplitude-low frequency of the input as well as making the device more useful for a range of frequency rather than just for a tonal frequency. The theoretical modelling of the softening and hardening mode is not the focus of this paper and the readers are referred to Kovacic and Brennan [22] to get the insight into the fundamental aspect of the Duffing oscillator. The content of this paper specifically focuses on the mechanism in producing the nonlinear hardening and softening mechanism, that is, involving mechanical domain only. The amount of electrical energy harvested by the mechanism is not the focus of this paper.

The content of the paper is organised as follows. The next section describes the measurement of vibration signals from different locations on a human body as the subject

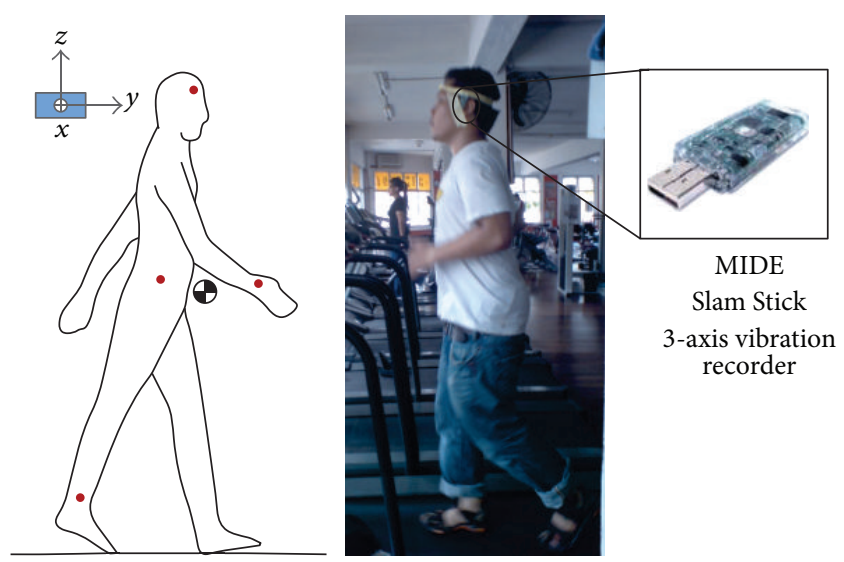

FIGURE 1: Experiment setup for measuring vibration signals from human motion. The spots on the left-hand side figure are the locations of the MIDE vibration data logger.

walks and runs at $5 \mathrm{~km} / \mathrm{h}$ and $9 \mathrm{~km} / \mathrm{h}$, respectively. This is followed by the principle of operation of the proposed mechanism. The following section describes the experimental investigation which describes the mechanism and the setup of the experiment which includes both quasi-static and dynamic measurements. The experimental results of the combined mode is presented and discussed in detail in the following section. This paper is then concluded with some final remarks.

\section{Measurement of Human Motion Signals}

A subject weighing $70 \mathrm{~kg}$ and with height of $170 \mathrm{~cm}$ volunteered in the measurement. The measurement of vibration signals from human motion was carried out when the subject was walking at $5 \mathrm{~km} / \mathrm{h}$ and running at $9 \mathrm{~km} / \mathrm{h}$ on a treadmill. The measurement was conducted at various locations on a human body as shown in Figure 1 using a MIDE Slam Stick vibration data logger. The data logger was able to measure the motion in $z$-axis (vertical), $y$-axis (fore and aft), and $x$-axis (lateral). The head, wrist, waist, and leg were chosen as the recording spots. These parts of human body were selected due to the convenient placement (or location) of some electronic gadgets. For example, a bluetooth head set and a cochlear implant are placed on the head, watches are strapped to the wrist, mobile phones are placed near the waist, while some sport performance monitoring devices are normally worn on the leg. The measurement of the vibration signal from the proposed locations is important especially when designing a mechanism to power an electronic gadget placed at the chosen location. This is particularly important since every location on human body produces different strength of the signal.

The MIDE vibration data logger was securely strapped at the chosen locations to minimize the relative motion between the data logger and the human body. In this measurement, the axes of oscillation shown in Figure 1 were assumed to remain unchanged and the rotation during the measurement was ignored.

Figures 2 and 3 show some of the measurement results in terms of time histories and their respective harmonics for the 


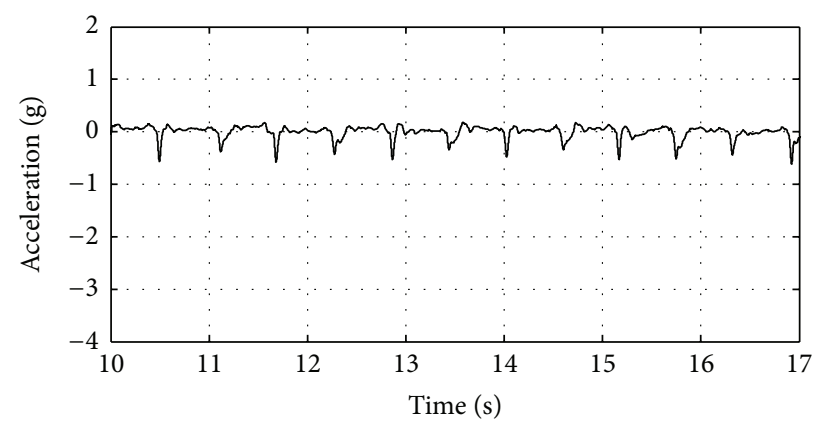

(a)

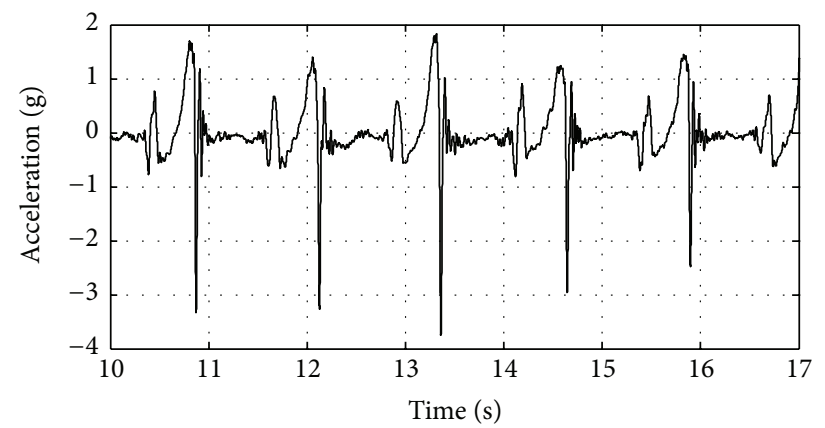

(c)

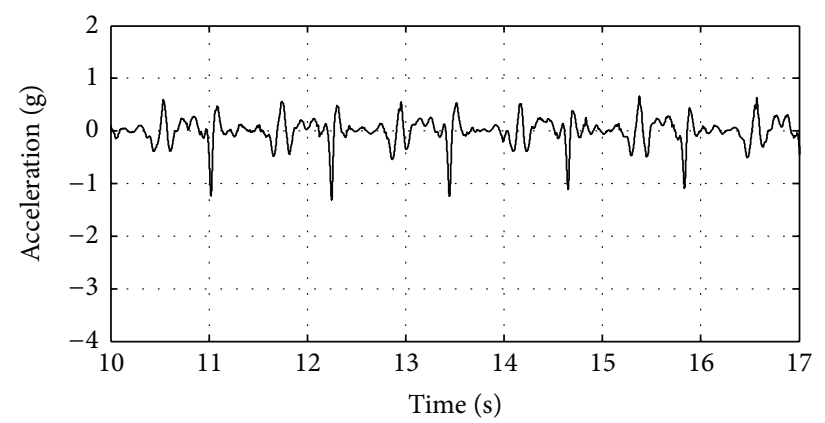

(e)

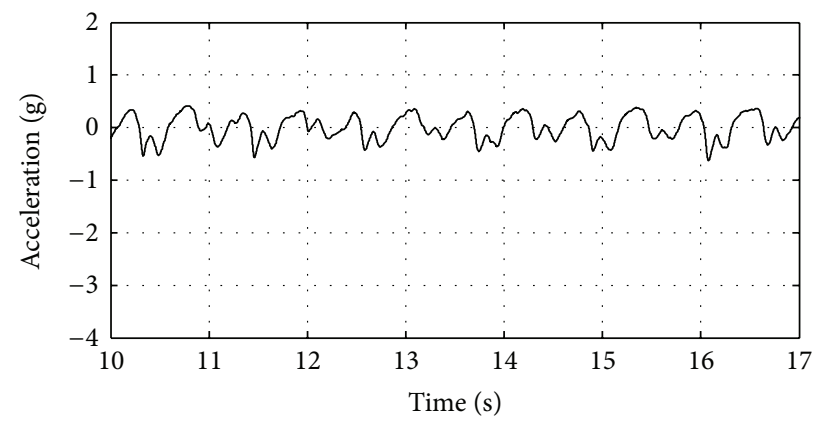

(g)

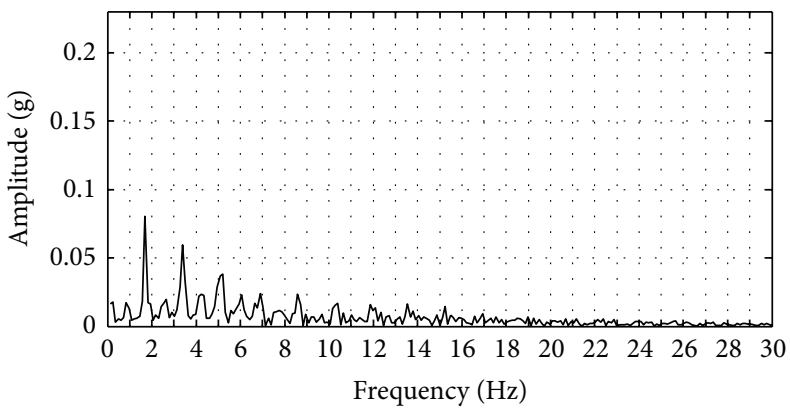

(b)

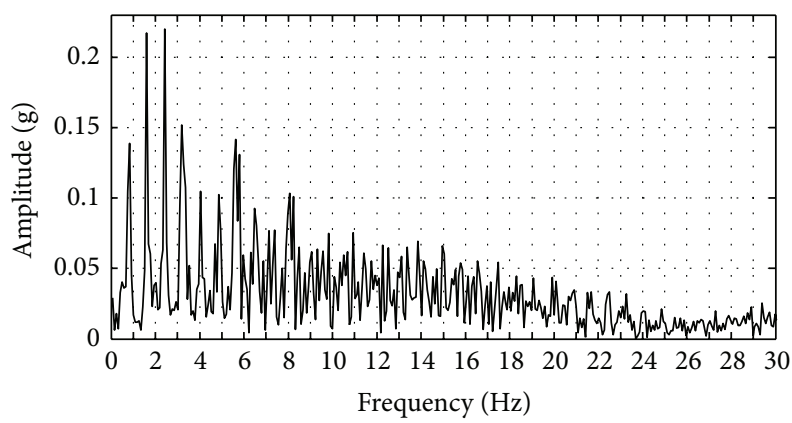

(d)

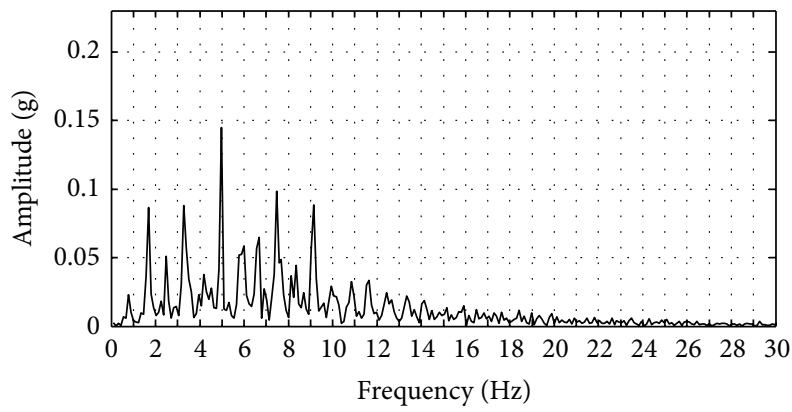

(f)

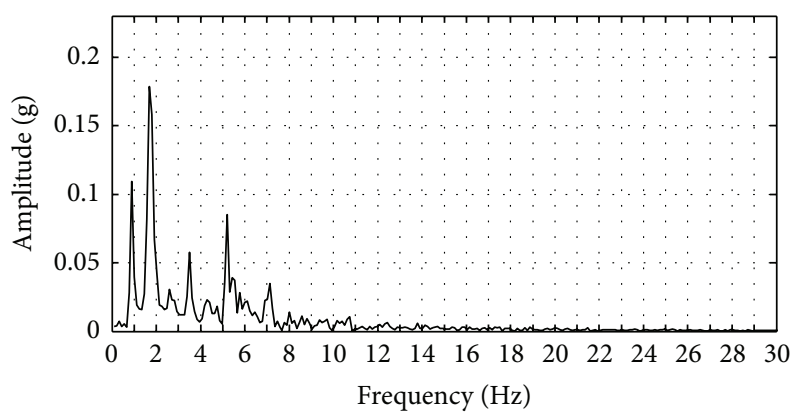

(h)

Figure 2: Time history ((a), (c), (e), (g)) and harmonics ((b), (d), (f), (h)) of human motion resulting from walking at $5 \mathrm{~km} / \mathrm{h}$. Vibration recorder was placed at the head ((a), (b)), leg ((c), (d)), waist ((e), (f)), and wrist ((g), (h)).

signals measured in the vertical direction (i.e., $z$-axis) only. From the measurement in all 3 axes, it can be concluded that the signals from the lateral direction ( $x$-axis) are, in general, the weakest. The strength of the signals in the vertical ( $z$-axis) and fore and aft ( $y$-axis) directions depends on the location and the speed of activity associated with it. For example, the strength of the signals recorded at the head and wrist shows a relatively similar strength in the vertical and fore-and-aft direction. They start to differ when the speed of the activity increases. The relatively similar strength of those directions is 


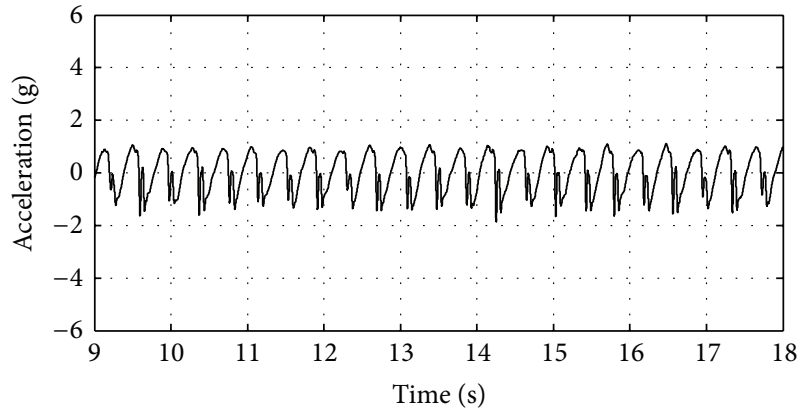

(a)

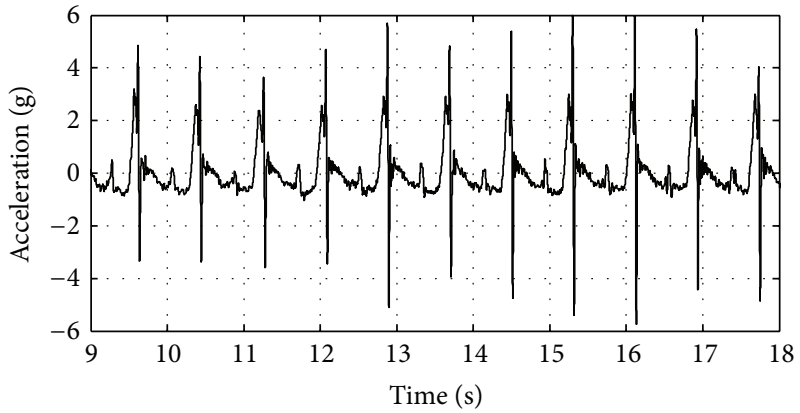

(c)

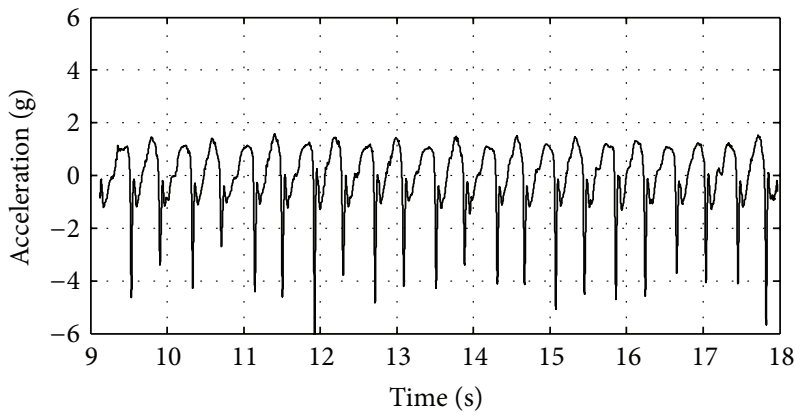

(e)

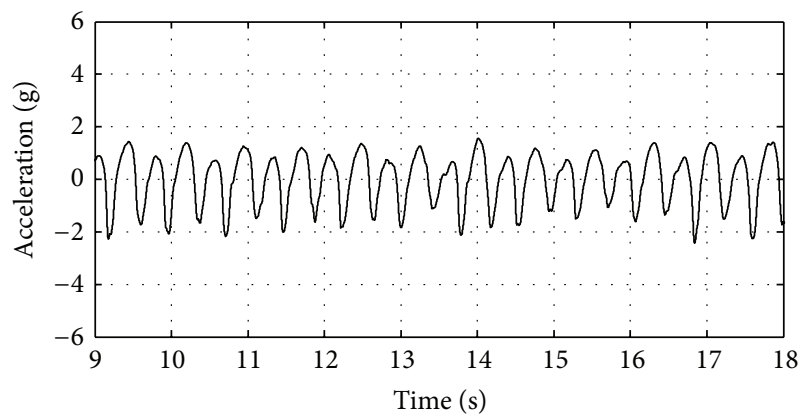

(g)

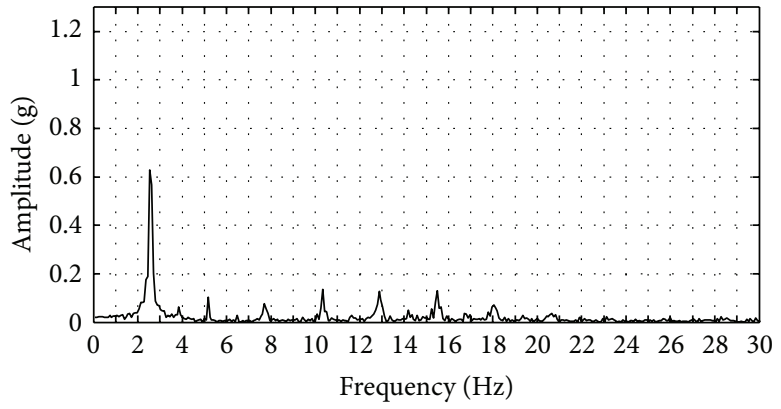

(b)

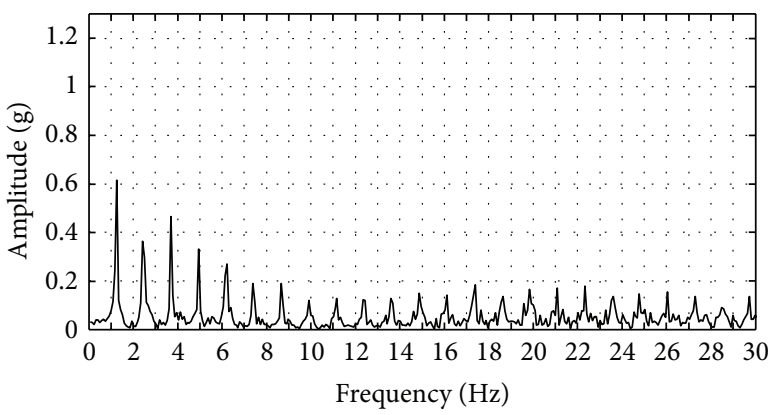

(d)

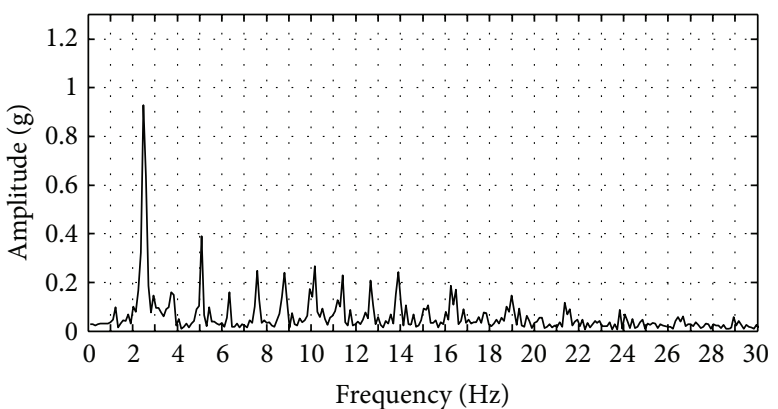

(f)

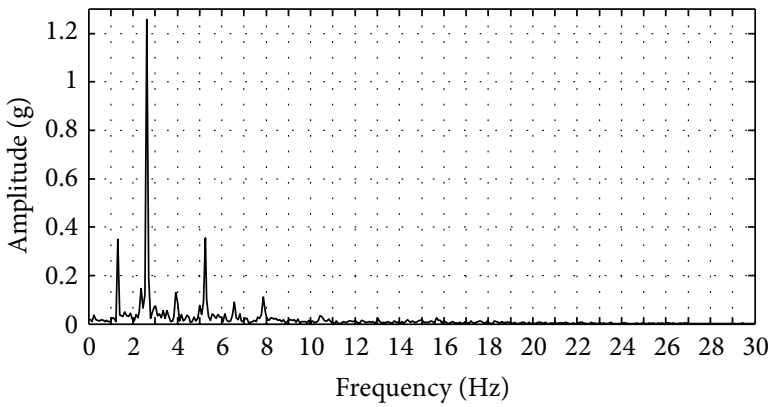

(h)

Figure 3: Time history ((a), (c), (e), (g)) and harmonics ((b), (d), (f), (h)) of human motion resulting from jogging at $9 \mathrm{~km} / \mathrm{h}$. Vibration recorder was placed at the head ((a), (b)), leg ((c), (d)), waist ((e), (f)), and wrist ((g), (h)).

due to the motion associated with the head and the wrist. As the subject walks and jogs, the head and the wrist undergo a rigid body motion. This effect is more visible when involving the leg motion in which the motion in the $y$-direction out classes the one in the $z$-direction when the subject is jogging at $9 \mathrm{~km} / \mathrm{h}$. The strength of the measured signals at the wrist, however, is more dominant in the $z$-direction then in the $y$ direction, suggesting the absence of the rigid body motion. 


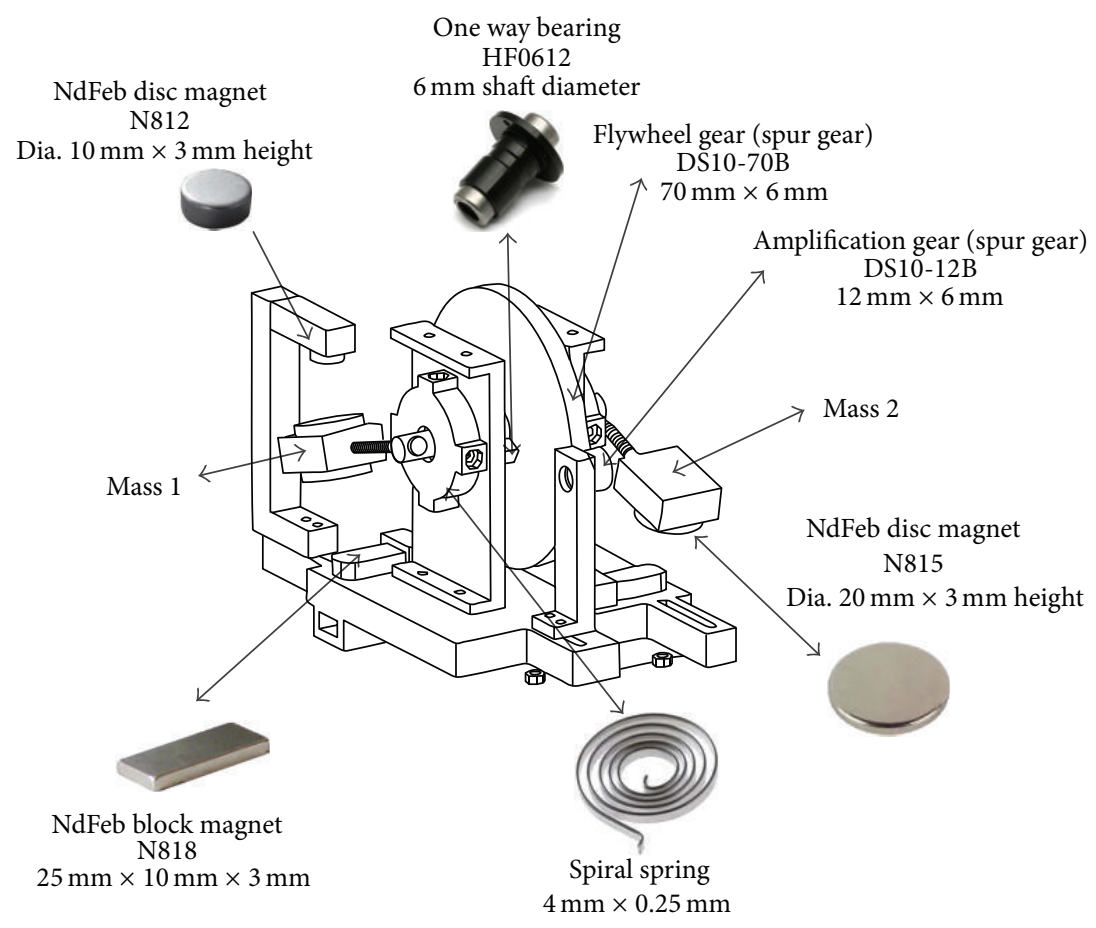

(a)

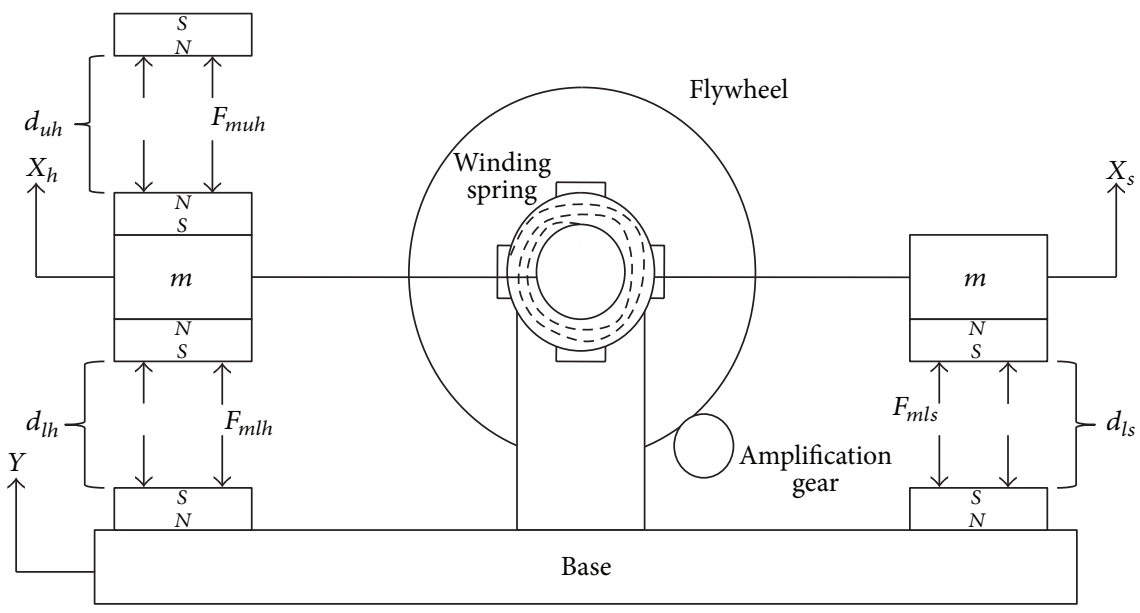

(b)

FIGURE 4: (a) Assembly drawing of the proposed mechanism and (b) the arrangement of the magnets. The left-hand side configuration consists of both top and bottom magnets while the right hand side configuration only consists of bottom magnet. $\left(d_{u h}, d_{l h}\right.$ and $d_{l s}$ are the distances between the magnets, $F_{m u h}, F_{m l h}$ and $F_{m l s}$ represent the magnetic restoring forces, $X_{h}$ is the displacement of the left hand side mass, $X_{s}$ is the displacement of the right hand side mass and $Y$ is input displacement of the base).

From this measurement, it can also be concluded that the signal from the head motion is the weakest while the leg motion is the strongest.

The strength of the motion dictates the energy distribution of the signal over the harmonics. The harmonics from the head and the wrist motion mostly lie in the region below $10 \mathrm{~Hz}$ and the harmonics from the waist motion spread in the region below $20 \mathrm{~Hz}$. The leg motion, on the other hand, produces harmonics up to $30 \mathrm{~Hz}$ as shown in Figures 2 and 3. These measured signals show the variability in the magnitude and the frequency content of different parts of human body motion. Designing an energy harvesting device which suits all locations involves a huge challenge as different location requires different needs to operate optimally.

\section{Principle of Working of the Proposed Mechanism}

The proposed mechanism utilizes the nonlinear hardening and softening stiffness to widen the bandwidth as well as to increase the low ambient frequency to a much higher output frequency. The proposed mechanism is shown in Figure 4(a) 
and the schematic is shown in Figure 4(b). The proposed mechanism consists of a shaft carrying a one way clutch bearing which is connected to the $70 \mathrm{~mm}$ diameter flywheel. The rotational motion of the shaft is governed by the stiffness provided by the winding spring. The shaft is then connected to two oscillating rods, each with a tip mass on each side of the flywheel. As the base moves up and down, the relative motions created by the masses drive the flywheel and thus rotate the $12 \mathrm{~mm}$ diameter amplification gear to result in the frequency-up conversion effect. Due to the one way clutch bearing, each oscillating mass rotates the flywheel in one direction only; that is, only half of the cycle of the oscillating masses rotates the flywheel.

The winding spring provides initial preset stiffness which can be first adjusted by winding up or unwinding the spring. This provides a tuning mechanism of the initial stiffness of the system. However, the stiffness of the winding spring is different for each direction of the motion. When the mass is moving in the direction of winding, the motion experiences a stiffer restoring force from the spring while when the masses are moving in the opposite direction, the motion experiences a softer restoring force from the spring. In this paper, the angle of winding is set at 180 degree for all cases.

The effective stiffness of the system is altered using the magnetic stiffness as configured in Figure 4. The right hand side mass experiences a hardening stiffness when moving downward and a softening stiffness during the upward motion (detailed discussion on stiffness behaviour is discussed in Section 5). On the other hand, the left-hand side mass experiences a hardening stiffness in both directions due to the repulsive magnetic stiffness on each side of the oscillating mass. The motions of these masses create a combined hardening and softening response which is aimed to perform better in terms of bandwidth widening and amplification of the human motion frequency and amplitude. The actual photo of the mechanism and the experimental setup is shown in Figure 5.

The behaviour of the mechanism is studied by changing the separation distance between each magnet. Three configurations are set to the mechanism as shown in Table 1. These configurations lead to different overall stiffness and responses which will be discussed in the later section.

\section{Experimental Investigation}

Two types of measurement were conducted in this section. The first was the quasi-static measurement of the force and the displacement of the masses. The second was the dynamic measurement of the acceleration of the masses.

In the quasi-static measurement, the total restoring force and the displacement of the masses were measured to study the stiffness characteristics of both masses independently and also to investigate the effect of combining both stiffness to the response of the amplification gear. The setup for the quasi-static measurement is shown in Figure 6. A force gauge and an angular decoder were attached to the mass which was driven by a shaker with large displacement amplitude at $0.5 \mathrm{~Hz}$. The force gauge was used to record the force while an angular decoder was used to measure the angle of rotation of

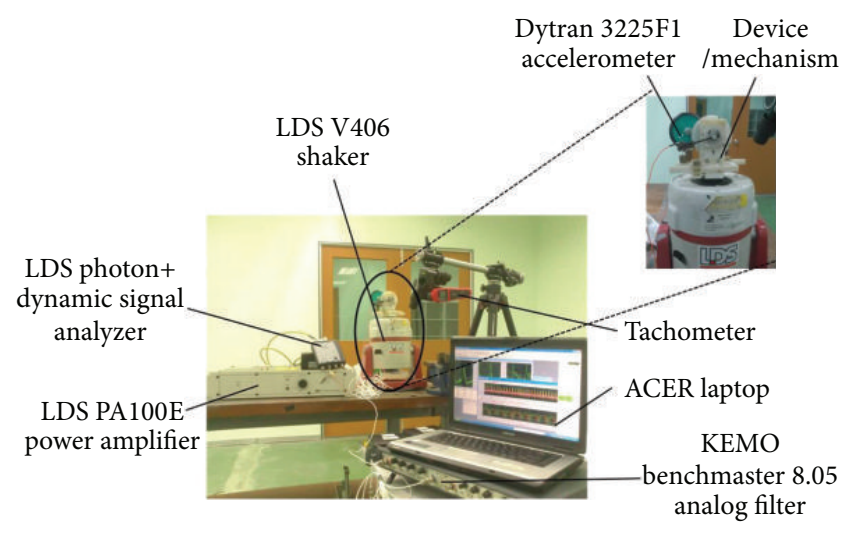

FIgURE 5: Experimental setup for dynamic measurement.

the masses. The displacement of the masses was then obtained using a small angle approximation. The masses were excited horizontally to minimize the effect of static deflection due to gravity.

In the dynamic measurement, the device was put onto a shaker and excited with pure sinusoidal wave. The frequency was increased from $1 \mathrm{~Hz}$ up to $15 \mathrm{~Hz}$ in $0.1 \mathrm{~Hz}$ steps and then decreased from $15 \mathrm{~Hz}$ down to $1 \mathrm{~Hz}$ with the same increment. The excitation from the shaker was maintained at $1 \mathrm{~g}$. The stiffness of the winding spring and the gap between the magnets were adjusted so that the natural frequency of the system was approximately around 5-6 Hz. Three accelerometers were used to capture the acceleration of the base and both masses. A tachometer was used to record the average rotational speed of the amplification gear which gives the resultant of the combined response between the softening and the hardening modes. The setup for the dynamic measurement is shown in Figure 7.

Another dynamic measurement was conducted using real human motion inputs shown in Figures 2 and 3. The measured inputs were decomposed into a sum of sinusoids to obtain the amplitude and phase of each harmonic. The signal was reconstructed by using less than 30 first harmonics which accounts for almost $90 \%$ of the total signal power. Figure 8 shows some of reconstructed signals together with their harmonics distribution. This shows that the signals are well reconstructed thus confirming that the strong energy of the signal lies in the first 30 harmonics. High frequency noise is also filtered out during the reconstruction of the signal.

The reconstructed signal was then fed into the shaker to drive the device. In the measurement of the average rotational speed of the amplification gear, the performance of the mechanism is compared by using three configuration setups as presented in Table 1. This is conducted for every location and activity of the measured signals presented in Figures 2 and 3.

\section{Results and Discussions}

The results from the quasi-static and dynamic measurements are presented in this section. Figures 9 and 10 show the forceand stiffness-displacement characteristics of the softening 


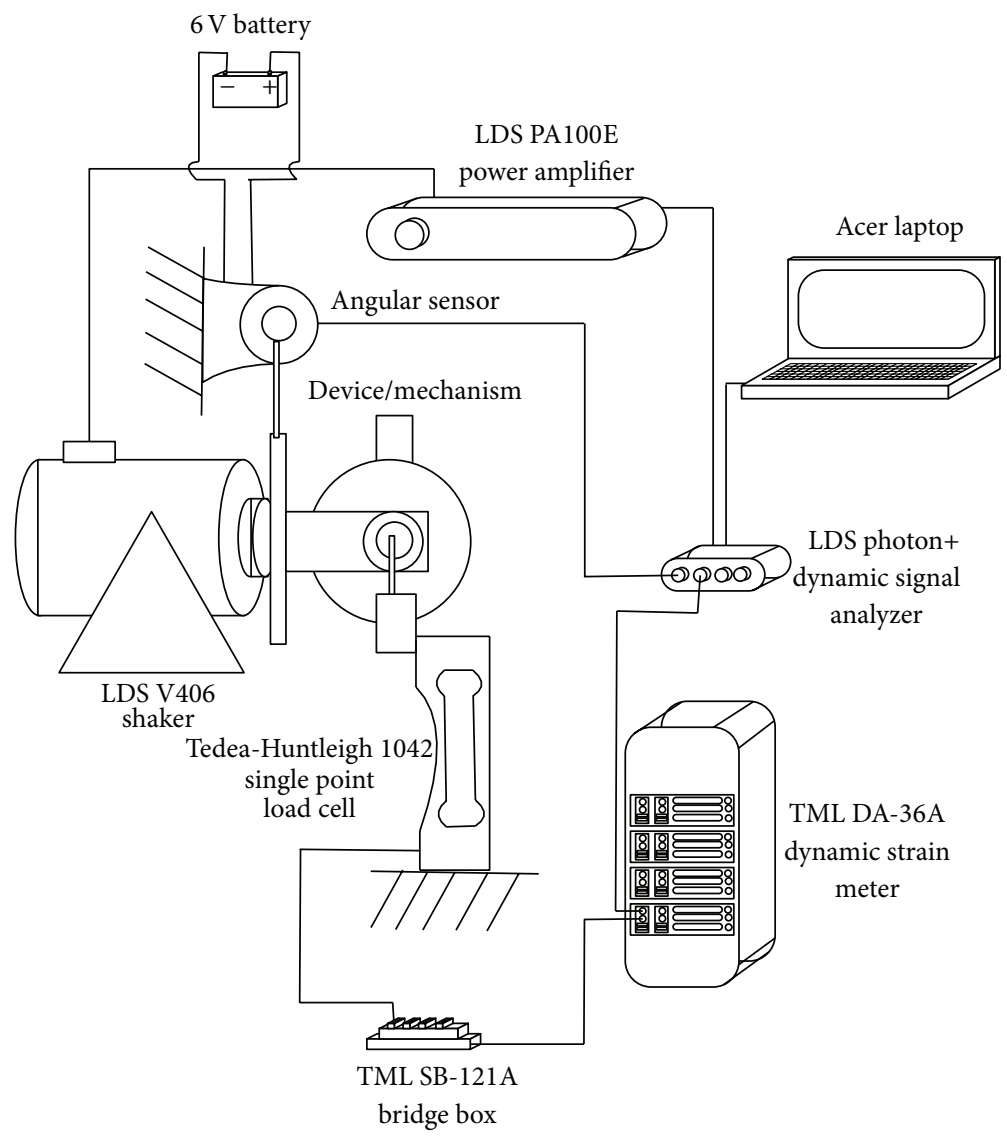

FIGURE 6: Setup for the quasi-static measurement.

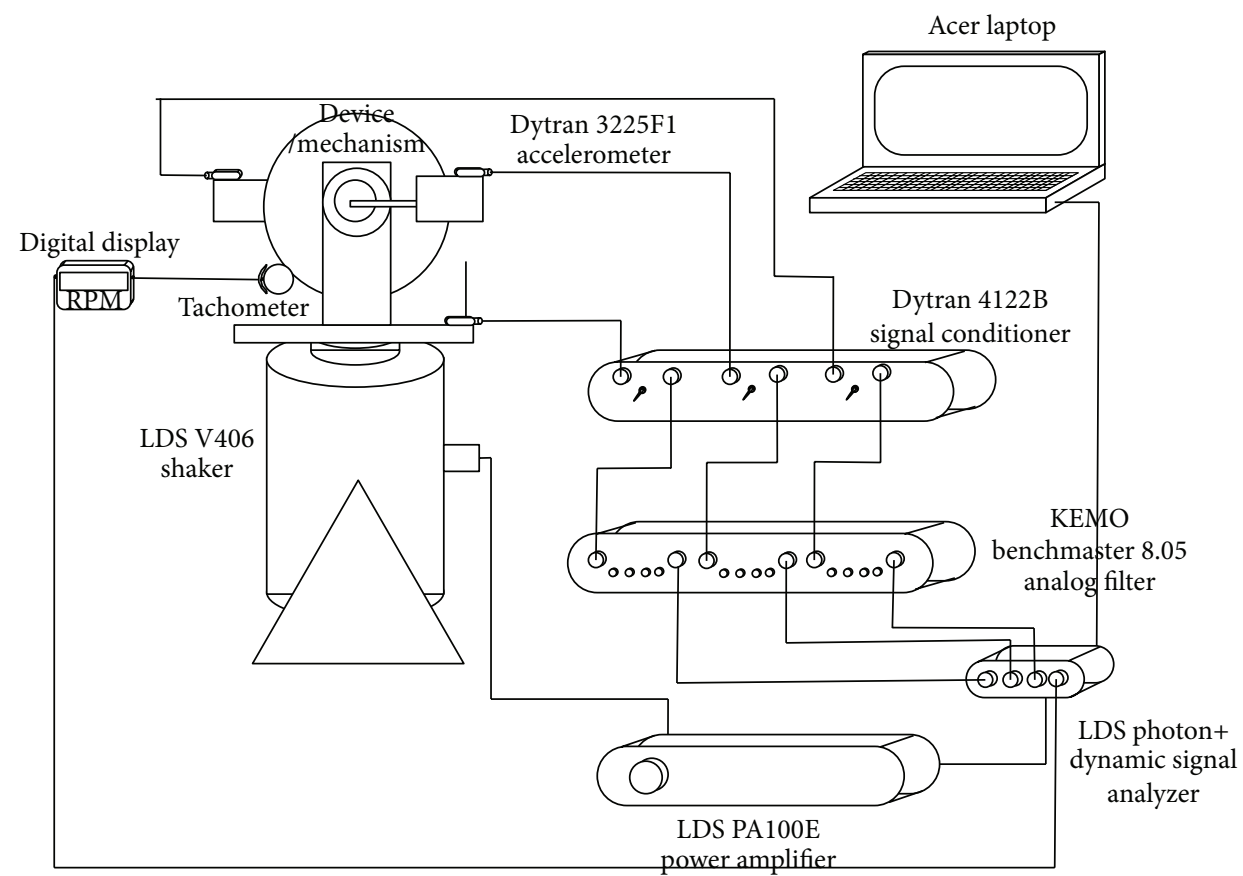

FIGURE 7: Setup for the dynamic measurement. 
TABLE 1: The gap between the top and bottom magnet with respect to the centre magnet.

\begin{tabular}{lccc}
\hline $\begin{array}{l}\text { Configuration } \\
\text { Magnet gap }\end{array}$ & $\begin{array}{c}\text { Softening configuration } \\
\text { Top gap, } d_{l s}(\mathrm{~mm})\end{array}$ & Top gap, $d_{u h}(\mathrm{~mm})$ & 30 \\
\hline Configuration 1 & 10 & 23 & 25 \\
Configuration 2 & 15 & 15 & 23 \\
Configuration 3 & 20 & 21 & Bottom gap, $d_{l h}(\mathrm{~mm})$ \\
\hline
\end{tabular}

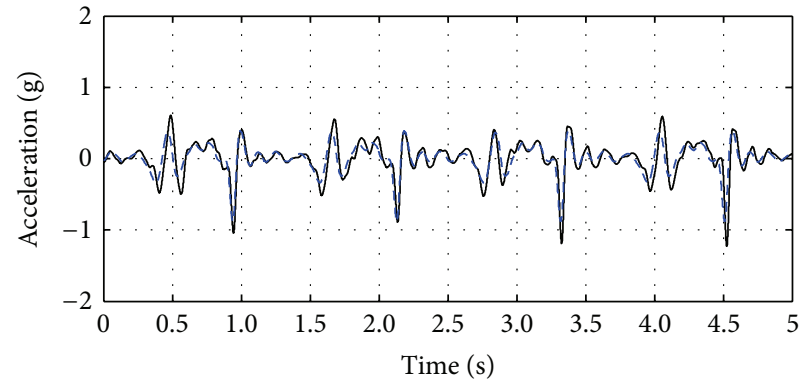

(a)

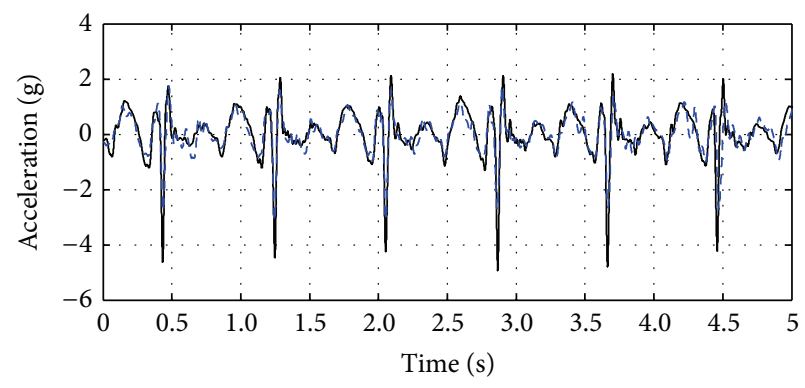

(c)

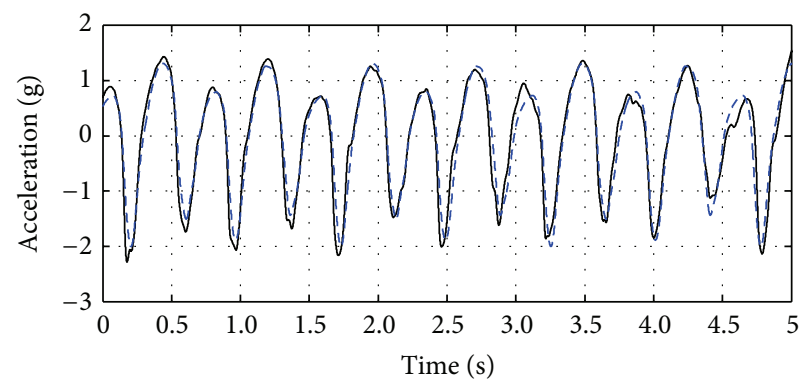

(e)

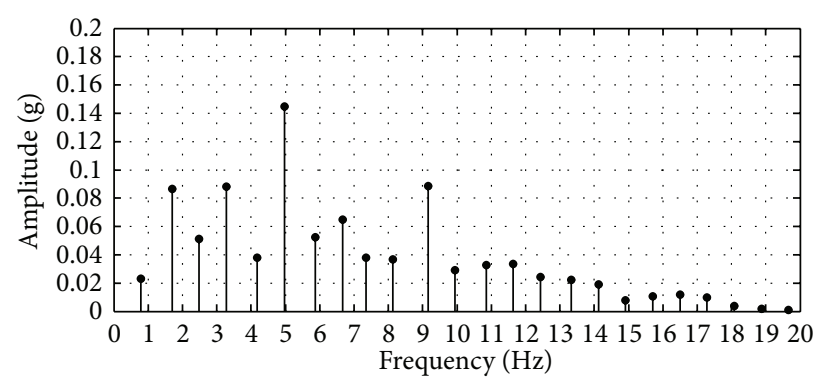

(b)

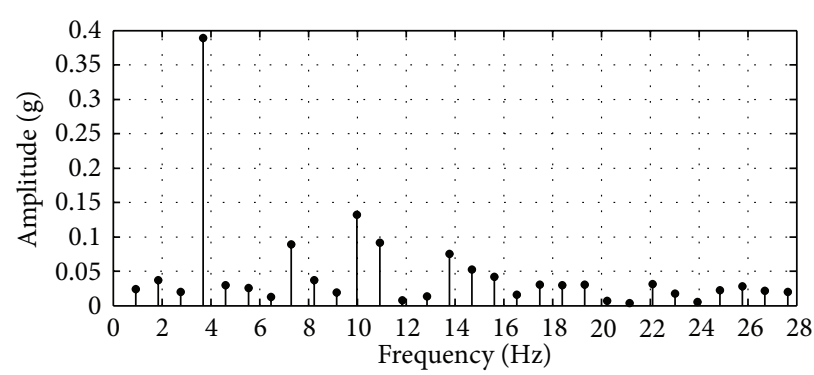

(d)

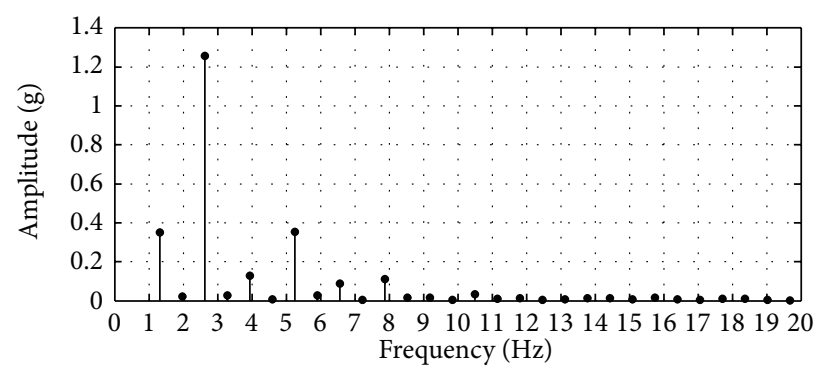

(f)

Figure 8: Time history comparison between the measured (solid black) and the reconstructed signals (dashed blue) ((a), (c), (e)) and the harmonic amplitude of the reconstructed signals ((b), (d), (f)). Signal at waist at $5 \mathrm{~km} / \mathrm{h}((\mathrm{a}),(\mathrm{b}))$ and leg at $9 \mathrm{~km} / \mathrm{h}((\mathrm{c})$, (d)) in $z$-axis and $y$-axis signal at wrist at $9 \mathrm{~km} / \mathrm{h}((\mathrm{e}),(\mathrm{f}))$.

and hardening modes, respectively. Figure 9 shows the forceand stiffness-displacement characteristics of the right hand side configuration of the proposed mechanism shown in Figure 4. This figure shows that the right-hand side mass experiences both softening and hardening restoring force during the motion. The hardening restoring force is due to the repulsive magnetic stiffness. This strong repulsive magnetic stiffness stores elastic potential energy during the downward motion (i.e., negative displacement) which is later used to throw the mass upward (i.e., positive displacement). Since the stiffness during the upward motion is governed by the relatively soft winding spring stiffness, it takes the mass to travel a longer distance for every unit of the restoring force for the winding spring to absorb the energy, thus resulting in a softening behaviour during the upward motion of the mass.

Differences can be noted in each three configurations. Decreasing the bottom magnet gap of the softening $d_{l s}$ increases the repulsive force between the centre and the bottom magnets. This increment in repulsive forces tends to increase the nonlinearity of the system, thus making the 


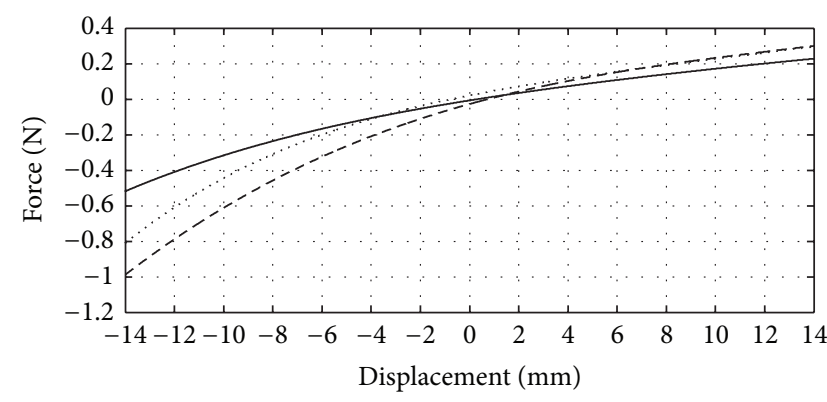

(a)

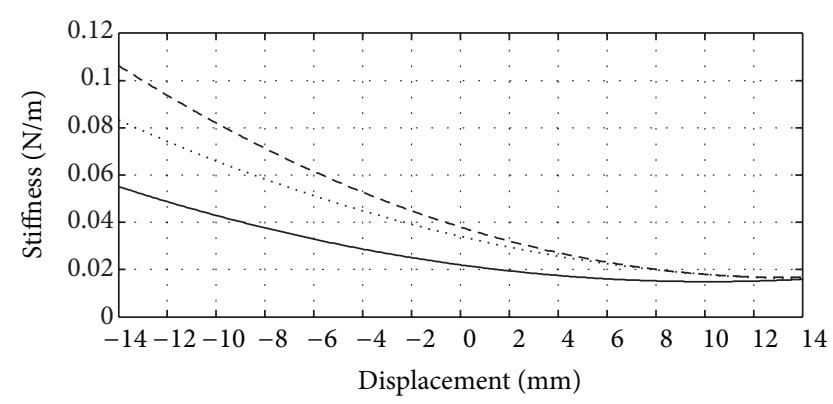

(b)

FIGURE 9: (a) Force against displacement and (b) stiffness against displacement curves for nonlinear softening configuration (right hand side configuration of Figure $4(\mathrm{~b}))$ for different gaps: $d_{l s}=20 \mathrm{~mm}$ (solid line), $d_{l s}=15 \mathrm{~mm}$ (dotted line), $d_{l s}=10 \mathrm{~mm}$ (dashed line).

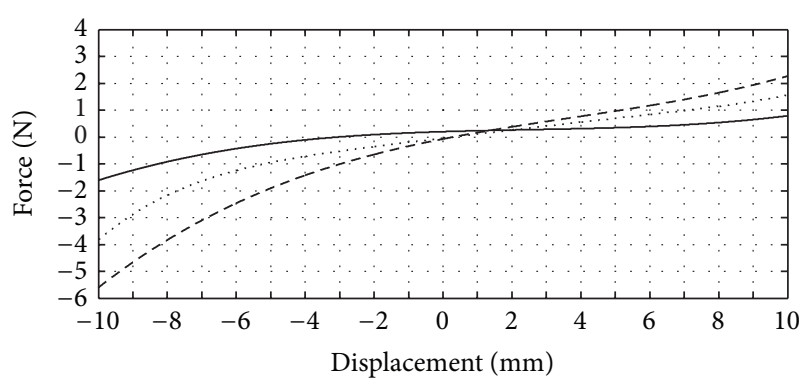

(a)

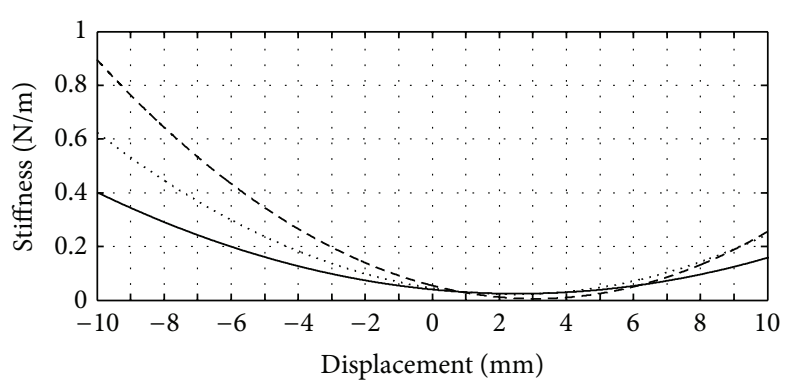

(b)

FIGURE 10: (a) Force against displacement and (b) stiffness against displacement curves for nonlinear hardening configuration (left hand side configuration of Figure 4(b)). Configuration $1, d_{u h}=30 \mathrm{~mm}$ and $d_{l h}=25 \mathrm{~mm}$ (solid line); Configuration $2, d_{u h}=23 \mathrm{~mm}$ and $d_{l h}=23 \mathrm{~mm}$ (dotted line); Configuration $3, d_{u h}=15 \mathrm{~mm}$ and $d_{l h}=21 \mathrm{~mm}$ (dashed line).

stiffness larger in the downward deflection. However, in the upward motion, since it is governed by the winding spring stiffness, the differences in all three configurations are small.

Figure 10 shows the force- and stiffness-displacement characteristics of the left hand side configuration shown in Figure 4(b). Three configurations are considered in the study as shown in Table 1. These three configurations are classified in terms of the gap between the top and bottom magnets with respect to the centre magnet. These gaps are chosen arbitrarily in order to show the differences. For each configuration, the upward (i.e., positive displacement) and the downward motion (i.e., negative displacement) curves show the decreasing function of displacement for a given force, which represents the nonlinear hardening characteristics. Note that in Figure 10, the force- and stiffness-displacement curves for each configuration are not symmetrical, with the downward motion part exhibiting stronger hardening characteristics than the upward motion part. The repulsive force of the top magnet is less than the bottom repulsive force due to the smaller gap in the bottom part than in the top part. The smaller the gap, the larger the force needed to displace the centre mass. Among the three configurations, Configuration 3 shows the largest restoring force when the mass is approaching its maximum displacement whereas the smallest restoring force at maximum deflection is recorded from Configuration 1.
The frequency response curves of hardening and softening modes are shown in Figures 11 and 12, respectively. These two figures were plotted independently in order to study the contribution from each mode. Figure 11 shows the hardening behaviour of the left-hand side stiffness. When the frequency is swept from $1 \mathrm{~Hz}$ to $15 \mathrm{~Hz}$, the response increases up to a maximum point where it suddenly drops to lower amplitude. On the other hand, when the frequency is swept from $15 \mathrm{~Hz}$ down to $1 \mathrm{~Hz}$, the response gradually increases until it reaches a point where it suddenly jumps up to larger amplitude. When the frequency is further decreased, the amplitude decreases as well converging to the response from the sweep-up frequency. The varying degree of nonlinearity is depicted by the jumpup and jump-down points. The higher the nonlinearity the higher the jump-up and jump-down frequencies are. This degree of nonlinearity is altered by adjusting the gap between the repulsive magnets shown in Figure 4(b); that is, the smaller the gap the larger the degree of the nonlinearity.

Figure 12 shows the softening behaviour of the right-hand side stiffness for different degrees of nonlinearity (i.e., gap). Similar to the hardening mode, the effect of the degree of the nonlinearity on the response is shown by the jump-up and jump-down points. However, in the softening mode, the larger the degree of nonlinearity is, the lower the jump frequencies are; that is, the response skews to the left instead of to the right. In this mode, the smaller the gap, the degree of 


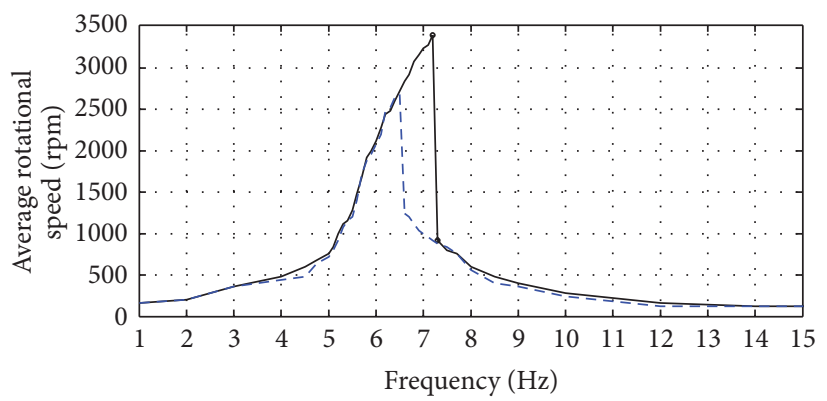

(a)

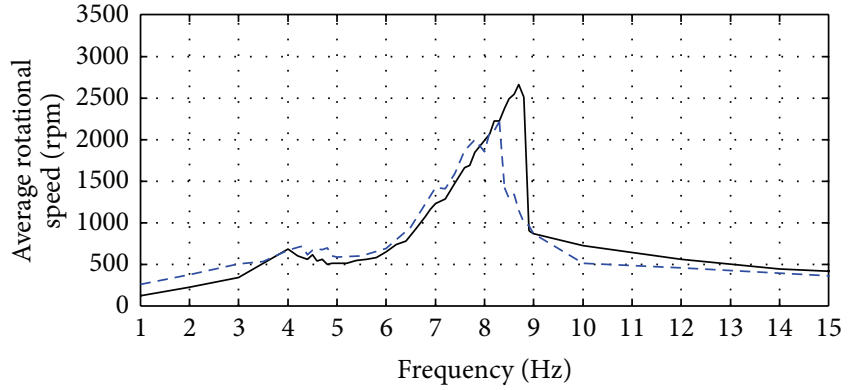

(b)

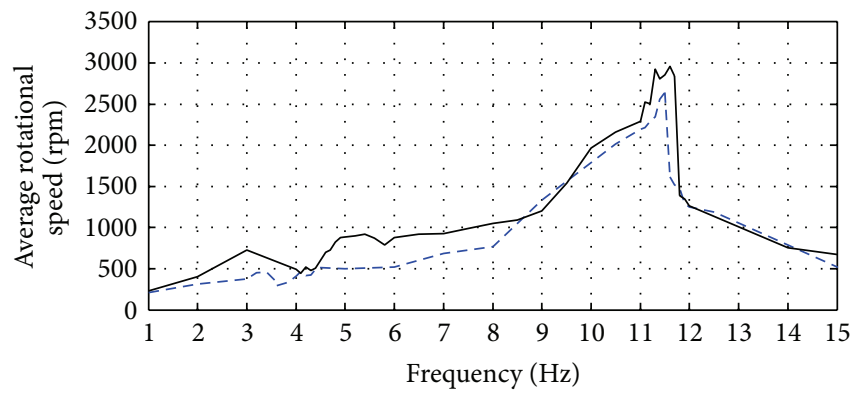

(c)

FIGURE 11: Frequency response curve of device with hardening configuration. Parameters setting of (a) $d_{u h}=30 \mathrm{~mm}$ and $d_{l h}=25 \mathrm{~mm}$, (b) $d_{u h}=23 \mathrm{~mm}$ and $d_{l h}=23 \mathrm{~mm}$, and (c) $d_{u h}=15 \mathrm{~mm}$ and $d_{l h}=21 \mathrm{~mm}$. Sweep up frequency (solid black) and sweep down frequency (dashed blue).

the softening behaviour of the stiffness increases. Decreasing the gap increases the stiffness during downward motion of the mass. For a given input, the amount of elastic energy stored for every unit displacement during the downward motion increases with the decrease in the gap. Hence by conservation of energy, the mass covers a large distance per unit force in the upward motion as the gap gets smaller.

Both Figures 11 and 12 show good characteristics of the softening and hardening modes for energy harvesting especially involving low-frequency and small-amplitude excitation. Both modes are able to widen the bandwidth in opposite direction. In particular, the softening mode can be useful when dealing with low frequency and small amplitude ambient motion in which it can amplify the amplitude of the excitation at low frequencies (i.e., jump-up point). This ability to amplify the amplitude of excitation is particularly important for harvesting energy from human body motion. This sudden jump in the amplitude is not possible in the linear resonant generator as the response of the linear generator increases and decreases monotonically with the excitation. On the other hand, the hardening mode is useful in amplifying the amplitude of the excitation (i.e., jump-up point) and widening the bandwidth in the higher frequency region (i.e., jump-down point).

Combining both nonlinear hardening and softening modes into a mechanism yields the response shown in Figure 13. Figure 13(a) shows the response of Configuration 1. In this configuration, both modes are tuned to operate very close to each other, resulting in an overlapped response which produces almost a flat response between $4 \mathrm{~Hz}$ and $7 \mathrm{~Hz}$. This results in a wider range of useful operating frequency of the system. In this configuration the decrease in the amplitude of one mode is compensated by the amplification of the amplitude from another mode resulting in a flat response. This is the effect of the response from the hardening mode in Figure 11(a) and the softening mode in Figure 12(a). At low frequencies, the softening mode contributes by amplifying the small amplitude to larger amplitude. When the response of the softening mode starts to decrease, the hardening mode contributes by driving up the amplitude of the response again. This kind of response is beneficial when dealing with a localized broad band excitation frequency.

As the nonlinearity of both modes increases, the responses start to separate apart in which the softening mode shifts to the left while the hardening mode moves to the right. This is shown in Figures 13(b) and 13(c) which corresponds to Configurations 2 and 3 presented in Table 1, respectively. In these two configurations, the decrease in the amplitude of one mode cannot be compensated by another mode since the two modes are moving away from each other and starting to behave independently. Under these conditions, Configurations 2 and 3 are behaving like a multimodal system. This kind of behaviour may be useful in applications involving a broadband excitation frequency.

The ability of the device to amplify the excitation frequency in terms of rotational speed is also shown in Figure 13. Taking Configuration 1 for example, at $5.4 \mathrm{~Hz}$ excitation, the rotational speed of the amplification gear rapidly increases from around $1700 \mathrm{rpm}$ to $2707 \mathrm{rpm}$ (equivalent to $45 \mathrm{~Hz}$ ). This is about 8.4 times increase in the excitation frequency 


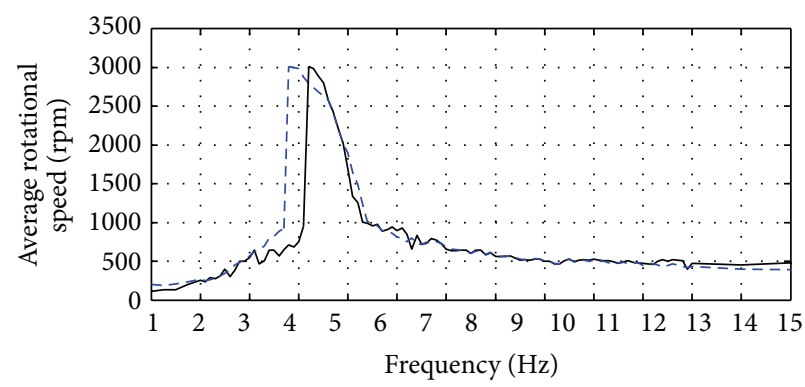

(a)

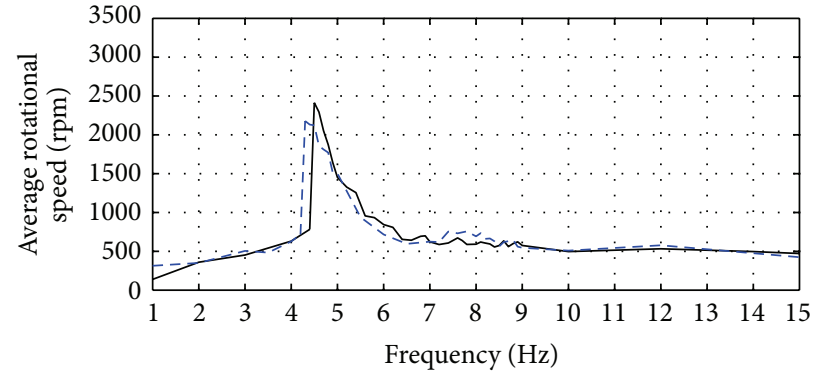

(b)

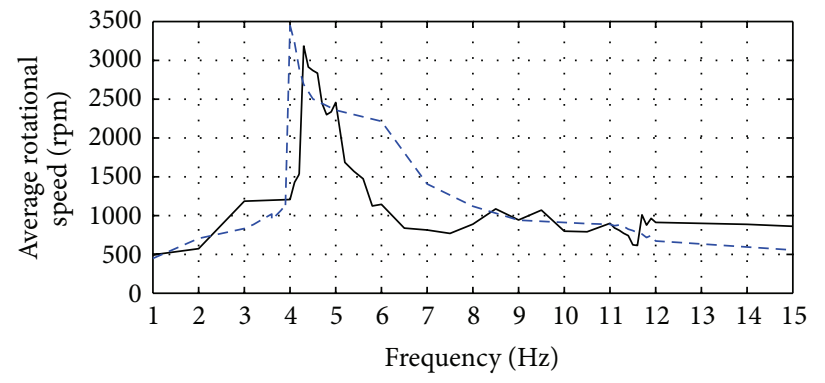

(c)

FIGURE 12: Frequency response curve with different bottom magnet separation distance $d_{l s}$. (a) $d_{l s}=25 \mathrm{~mm}$, (b) $d_{l s}=15 \mathrm{~mm}$, and (c) $d_{l s}=10 \mathrm{~mm}$. Sweep up frequency (solid black) and sweep down frequency (dashed blue).

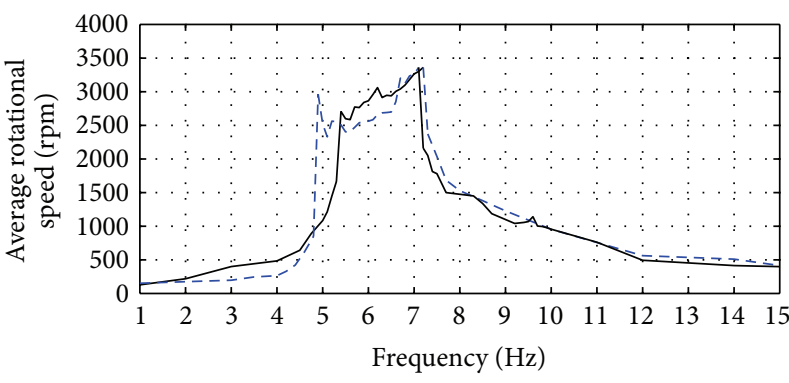

(a)

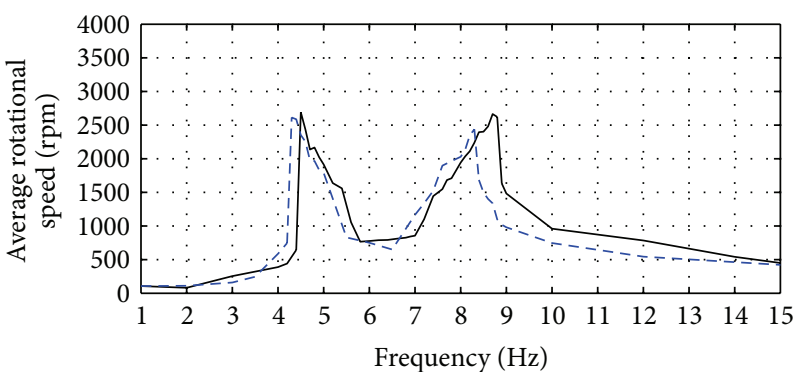

(b)

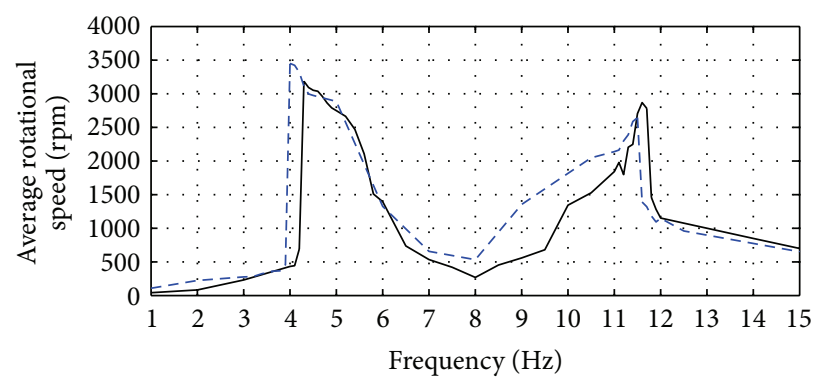

(c)

FIGURE 13: Frequency response curve for the combined hardening and softening modes. Sweep up frequency (solid black) and sweep down frequency (dashed blue). (a) Configuration 1, (b) Configuration 2, and (c) Configuration 3.

and this is mainly due to the jump-up phenomena of the softening mode described in Figure 12. The rotational speed increases with the excitation frequency up to $7.2 \mathrm{~Hz}$ with $3362 \mathrm{rpm}(56 \mathrm{~Hz})$ rotational speed of the amplification gear. This accounts for 7.8 times increase in output frequency compared to the excitation frequency. However, if the frequency is further increased, the response drops significantly due to the jump-down phenomenon in the hardening mode as presented in Figure 11.

The ability of the device to perform under a broadband frequency is studied by exciting the device with measured signals from human body motion. The signals were taken from 
TABLE 2: Average rotational speed resulting from the measured input. The bold values are the fastest rotational speed for each location and activity.

\begin{tabular}{|c|c|c|c|c|}
\hline \multicolumn{2}{|c|}{ Signal location/activity } & \multicolumn{3}{|c|}{$\begin{array}{l}\text { Average rotational speed [RPM] } \\
\text { Combined mode configuration with } 180 \text { degree angle of twist }\end{array}$} \\
\hline & & Configuration 1 & Configuration 2 & Configuration 3 \\
\hline \multirow{2}{*}{ Head } & Walking & 360.1 & 335.0 & 286.3 \\
\hline & Running & 680.4 & 886.8 & 963.6 \\
\hline \multirow{2}{*}{ Wrist } & Walking & 714.2 & 781.4 & 621.8 \\
\hline & Running & 1988.0 & 2029.0 & 1956.0 \\
\hline \multirow{2}{*}{ Waist } & Walking & 652.4 & 585.4 & 637.3 \\
\hline & Running & 1740.0 & 1147.0 & 1322.0 \\
\hline \multirow{2}{*}{ Leg } & Walking & 1396.0 & 931.7 & 1351.0 \\
\hline & Running & 2280.0 & 1295.0 & 1871.0 \\
\hline
\end{tabular}

different locations on the human body, that is, head, leg, waist, and wrist. The measured signals were reconstructed and fed to the shaker. The performance of the device is investigated by comparing the response of the three configurations of the combined mode.

Table 2 presents the average rotational speed of the amplification gear for the combined mode when excited with the measured signals from the vertical axis (i.e., $z$ axis) as presented in Figures 2 and 3. Note that, the three configurations used are not the optimal configuration. The aim is mainly to operate the device below $10 \mathrm{~Hz}$ and to investigate the feasibility of such device in harvesting energy from human body motion. It can be seen in Table 2 that the largest average rotational speed of the amplification gear depends on the setup of device (i.e., configuration), activity, and the location of the measured signal. However, Configuration 1 performs better compared to other two configurations especially when involving strong signals coming from the waist and the leg. It is thought that the strong harmonics are produced in the effective region of operation of Configuration 1 thus contributing to the large rotational speed. Table 2 also shows that the combined mode with separated hardening and softening modes as shown in Figures 13(b) and 13(c) is also beneficial especially when excited with the signals from the wrist and head motion.

\section{Conclusion}

The paper has presented experimental results for the combined nonlinear softening and hardening mode which is capable of amplifying the low ambient frequency as well as widening the bandwidth. The human motion signals were acquired from different locations of a human body. These locations were at the head, leg, wrist, and waist. The measurement revealed that the strong harmonics were found in the region below $30 \mathrm{~Hz}$ while the energy-rich harmonics were mostly found in the region below $10 \mathrm{~Hz}$.

A mechanism utilizing both softening and hardening modes was proposed in this study. This mechanism was aimed at amplifying the low human motion frequency to a higher output frequency as well as to widen the bandwidth so that it is useful for a wider range of excitation frequency.
The proposed mechanism converted the translational motion of the input to the output rotational motion. The mechanism consisted of two oscillating masses connected to a one way clutch bearing through a rigid rod. The clutch was attached to a flywheel which was coupled to a small amplification gear. The motions of the masses rotate the flywheel which then rotates the amplification gear. The softening mode was aimed at increasing the amplitude of the oscillating masses thus resulting in a faster rotation of the flywheel while the hardening mode was aimed at widening the bandwidth. While the individual modes performed well in their jump frequency regions, the combined mode performed better and was able to increase the rotational speed of the amplification gear up to 8 times. In terms of the bandwidth widening, the combined mode outperformed the individual modes. This useful frequency band was obtained by sweeping up the excitation frequency and could be better if the excitation frequency was swept down since the jump-down frequency of the softening mode occurred at a much lower frequency.

The performance of the device under a broadband input was investigated by exciting the device with the measured signals from the human body motion. These signals were reconstructed and fed into the shaker. The overlapped response of the combined mode performed better especially when the harmonics of the measured signals lie in the effective operating region of Configuration 1. However, the other two configurations which produce nonoverlapped responses also performed better in some cases. From this study, it can be seen that it is important to have the knowledge of the excitation. If the harmonics of the excitation are concentrated in narrow frequency band thus the hardening and the softening modes must be tuned to produce an overlapped response. However, when the harmonics are not concentrated in a narrow frequency band and are distributed across a range of frequency, the two modes must be tuned so that their responses are well separated. This opens a new opportunity for future research on the optimal tuning technique of such mechanism.

\section{Conflict of Interests}

The authors declare that there is no conflict of interests regarding the publication of this paper. 


\section{Acknowledgments}

The authors would like to acknowledge the financial support provided by the Ministry of Higher Education Malaysia and Universiti Teknikal Malaysia Melaka through Fundamental Research Grant Scheme FRGS/2010/FKM/TK03/12/F00100.

\section{References}

[1] L. Tang, Y. Yang, and C. K. Soh, "Toward broadband vibrationbased energy harvesting," Journal of Intelligent Material Systems and Structures, vol. 21, no. 18, pp. 1867-1897, 2010.

[2] C. Eichhorn, F. Goldschmidtboeing, and P. Woias, "Bidirectional frequency tuning of a piezoelectric energy converter based on a cantilever beam," Journal of Micromechanics and Microengineering, vol. 19, no. 9, Article ID 094006, 2009.

[3] D. Zhu, S. Roberts, J. Tudor, and S. Beeby, "Closed loop frequency tuning of a vibration-based micro generator," in Proceedings of PowerMEMS, pp. 229-232, 2008.

[4] I. Sari, T. Balkan, and H. Kulah, "An electromagnetic micro energy harvester based on an array of parylene cantilevers," Journal of Micromechanics and Microengineering, vol. 19, no. 10, pp. 105023-1-105023-13, 2009.

[5] S. M. Shahruz, "Design of mechanical band-pass filters for energy scavenging," Journal of Sound and Vibration, vol. 292, no. 3-5, pp. 987-998, 2006.

[6] H. Xue, Y. Hu, and Q.-M. Wang, "Broadband piezoelectric energy harvesting devices using multiple bimorphs with different operating frequencies," IEEE Transactions on Ultrasonics, Ferroelectrics, and Frequency Control, vol. 55, no. 9, pp. 21042108, 2008.

[7] R. Ramlan, M. J. Brennan, B. R. Mace, and I. Kovacic, "Potential benefits of a non-linear stiffness in an energy harvesting device," Nonlinear Dynamics, vol. 59, no. 4, pp. 545-558, 2010.

[8] D. D. Quinn, A. L. Triplett, L. A. Bergman, and A. F. Vakakis, "Comparing linear and essentially nonlinear vibration-based energy harvesting," Journal of Vibration and Acoustics, Transactions of the ASME, vol. 133, no. 1, Article ID 011001, 2011.

[9] B. P. Mann and N. D. Sims, "Energy harvesting from the nonlinear oscillations of magnetic levitation," Journal of Sound and Vibration, vol. 319, no. 1-2, pp. 515-530, 2009.

[10] D. A. W. Barton, S. G. Burrow, and L. R. Clare, "Energy harvesting from vibrations with a nonlinear oscillator," Journal of Vibration and Acoustics, vol. 132, no. 2, Article ID 021009, 2010.

[11] R. Ramlan, M. J. Brennan, B. R. Mace, and S. G. Burrow, "On the performance of a dual-mode non-linear vibration energy harvesting device," Journal of Intelligent Material Systems and Structures, vol. 23, no. 13, pp. 1423-1432, 2012.

[12] G. Litak, M. I. Friswell, and S. Adhikari, "Magnetopiezoelastic energy harvesting driven by random excitations," Applied Physics Letters, vol. 96, no. 21, Article ID 214103, 2010.

[13] C. R. McInnes, D. G. Gorman, and M. P. Cartmell, "Enhanced vibrational energy harvesting using nonlinear stochastic resonance," Journal of Sound and Vibration, vol. 318, no. 4-5, pp. 655662, 2008.

[14] A. Cammarano, S. G. Burrow, and D. A. W. Barton, "Modelling and experimental characterization of an energy harvester with bi-stable compliance characteristics," Proceedings of the Institution of Mechanical Engineers I: Journal of Systems and Control Engineering, vol. 225, no. 4, pp. 475-484, 2011.
[15] A. Erturk and D. J. Inman, "Broadband piezoelectric power generation on high-energy orbits of the bistable Duffing oscillator with electromechanical coupling," Journal of Sound and Vibration, vol. 330, no. 10, pp. 2339-2353, 2011.

[16] S. C. Stanton, C. C. McGehee, and B. P. Mann, "Reversible hysteresis for broadband magnetopiezoelastic energy harvesting," Applied Physics Letters, vol. 95, no. 17, Article ID 174103, 2009.

[17] S. D. Nguyen and E. Halvorsen, "Nonlinear springs for bandwidth-tolerant vibration energy harvesting," Journal of Microelectromechanical Systems, vol. 20, no. 6, Article ID 6062373, pp. 1225-1227, 2011.

[18] G. Zhu, P. Bai, J. Chen, and Z. Lin Wang, "Power-generating shoe insole based on tribo electric nanogenerators for selfpowered consumer electronics," Nano Energy, vol. 2, no. 5, pp. 688-692, 2013.

[19] P. Pillatsch, E. M. Yeatman, and A. S. Holmes, "A piezoelectric frequency up-converting energy harvester with rotating proof mass for human body applications," Sensors and Actuators A: Physical, vol. 206, pp. 178-185, 2014.

[20] S. Wei, H. Hu, and S. He, "Modeling and experimental investigation of an impact-driven piezoelectric energy har vester from human motion," Smart Materials and Structures, vol. 22, no. 10, Article ID 105020, 2013.

[21] L. Gu and C. Livermore, "Impact-driven, frequency upconverting coupled vibration energy harvesting device for low frequency operation," Smart Materials and Structures, vol. 20, no. 4, Article ID 045004, pp. 045004-1-045004-10, 2011.

[22] I. Kovacic and M. J. Brennan, The Duffing Equation: Nonlinear Oscillators and Their Behaviour, John Wiley and Sons, Chichester, UK, 2011. 

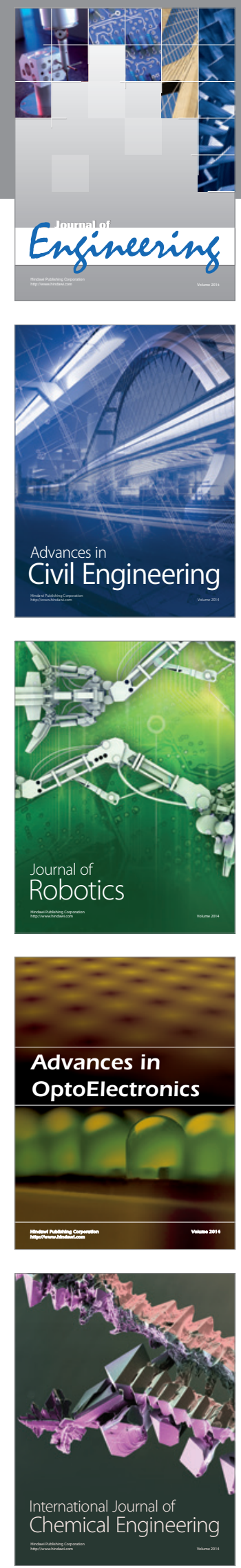

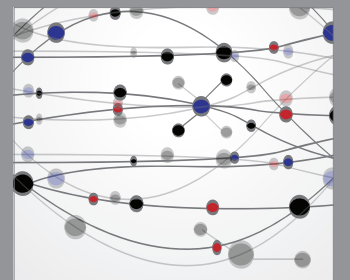

The Scientific World Journal
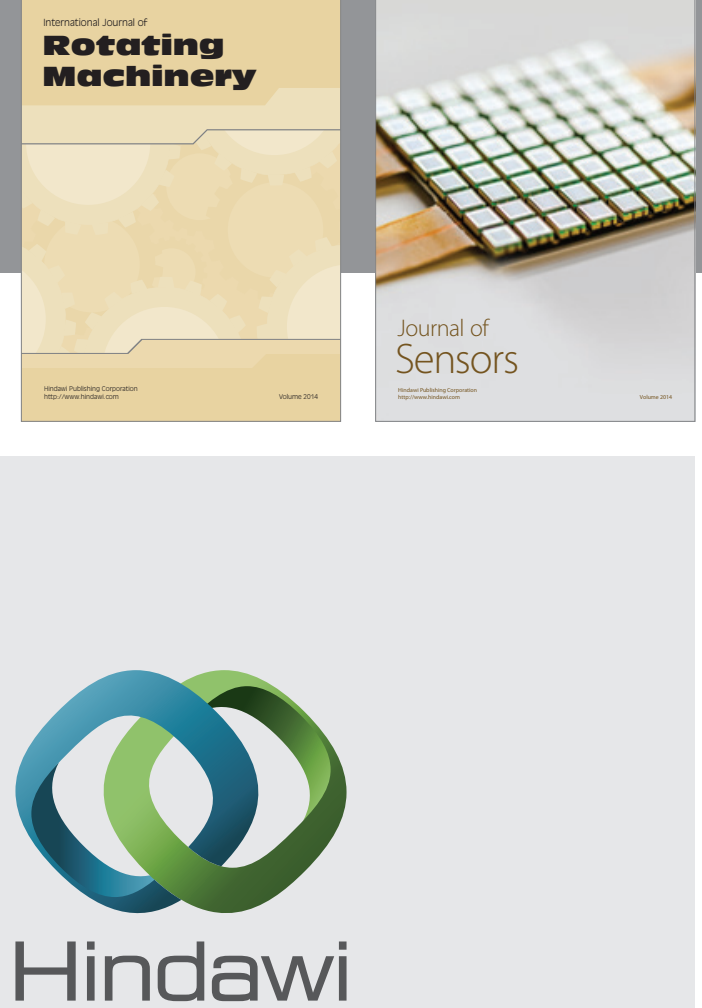

Submit your manuscripts at http://www.hindawi.com
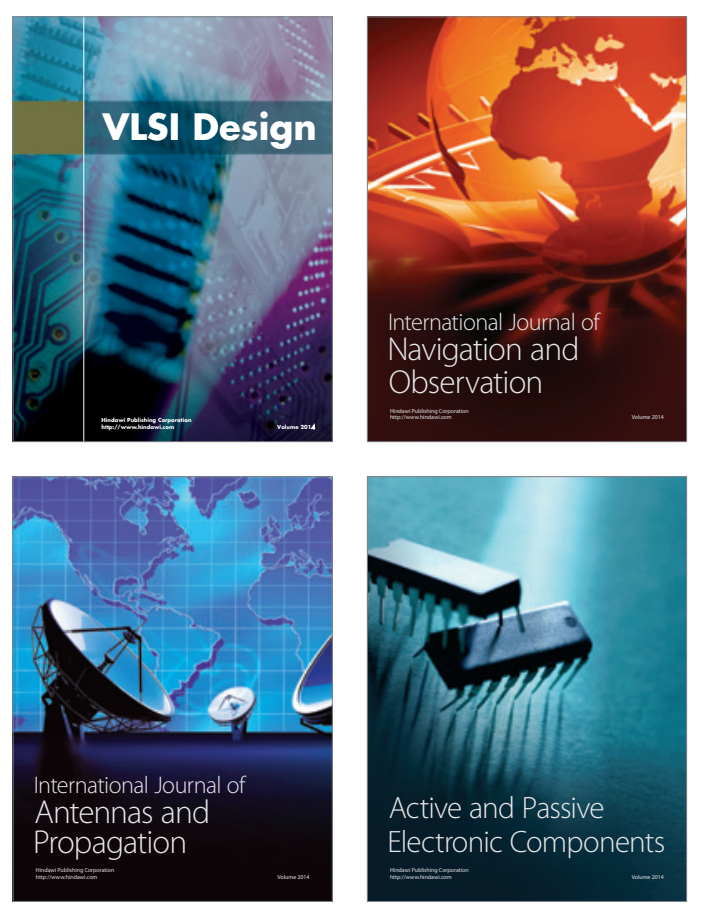
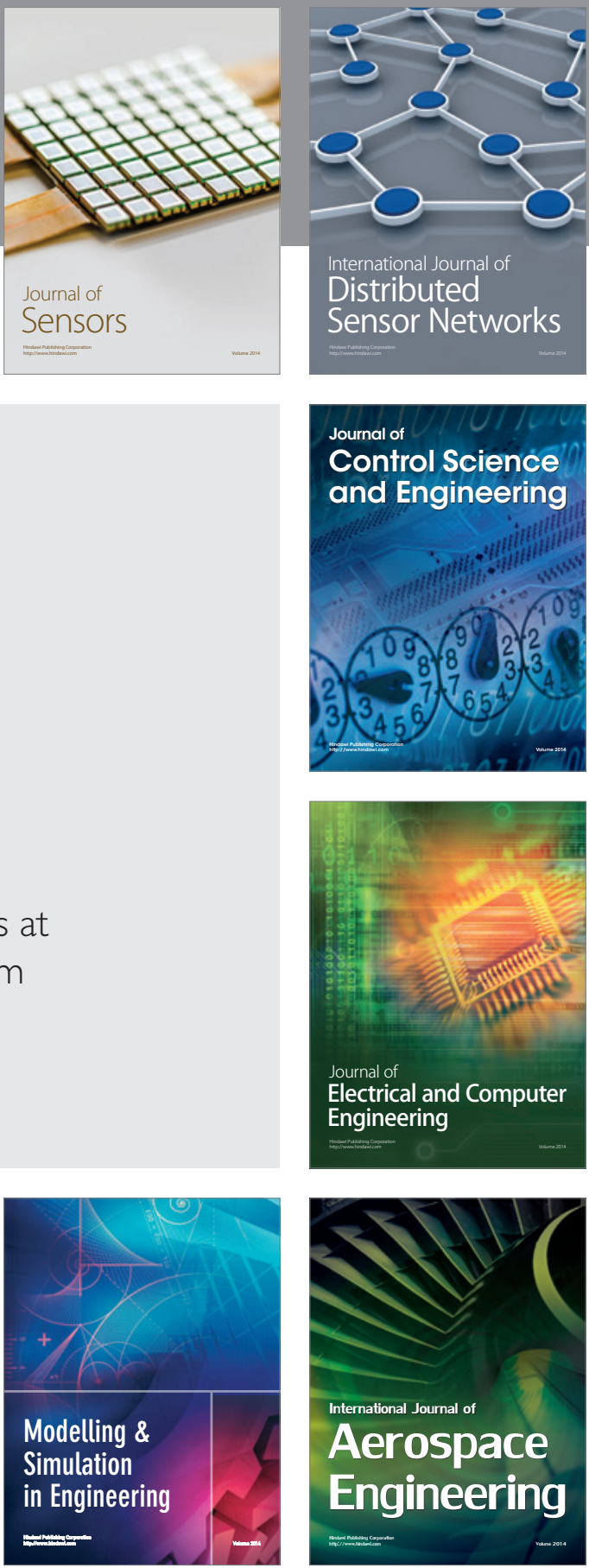

Journal of

Control Science

and Engineering
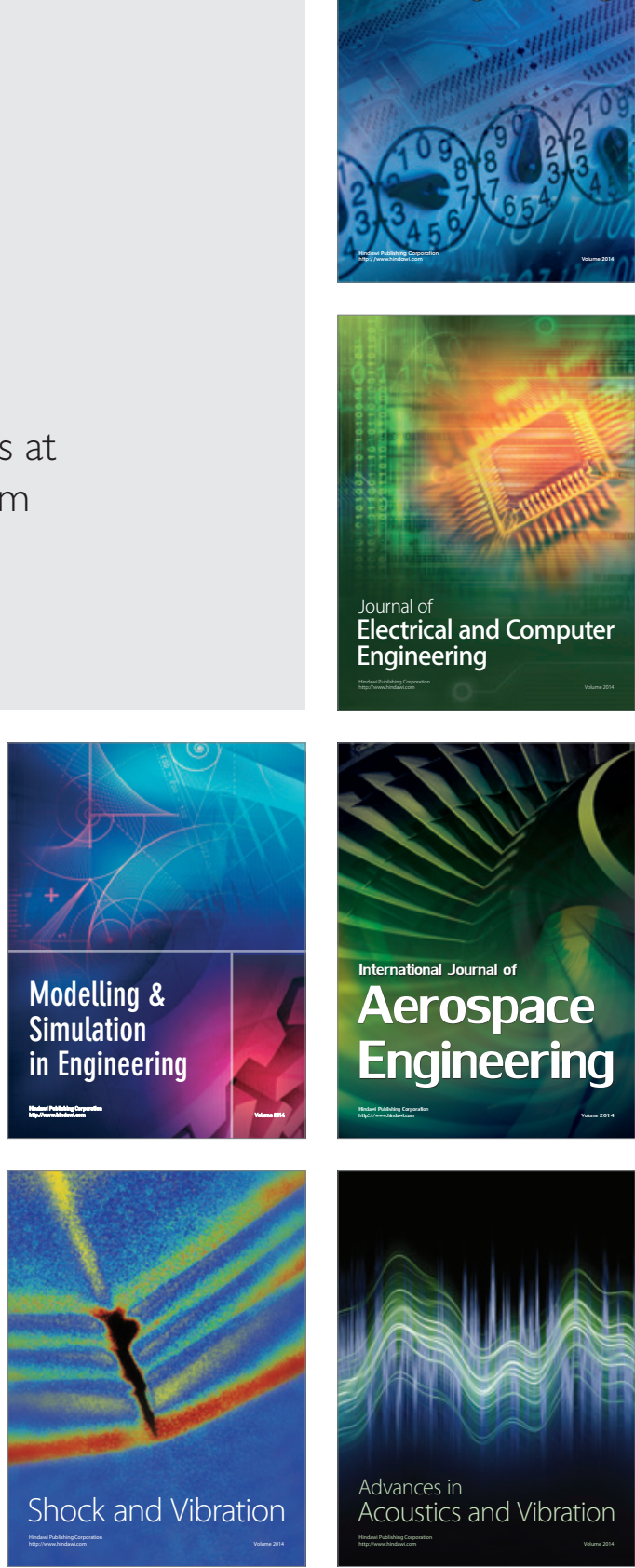